04

\title{
Сверхширокополосная антенна для регистрации радиоизлучения в начальной фазе высоковольтного лабораторного атмосферного разряда
}

\author{
(C) И.С. Байдин, А.В. Огинов, Е.В. Паркевич \\ Физический институт им. П.Н. Лебедева РАН, \\ 119991, Москва, Россия \\ e-mail: ivanbaidin@lebedev.ru \\ Поступило в Редакцию 26 января 2021 г. \\ В окончательной редакции 28 июня 2021 г. \\ Принято к публикации 11 июля 2021 г.
}

\begin{abstract}
Представлена малогабаритная сверхширокополосная антенна, разработанная для применения в экспериментах по исследованию радиоизлучения, генерирующегося в начальной фазе искрового разряда. Рассмотрен плоский тип антенн на основе расширяющейся щели (антенна Вивальди). Проведены моделирование и расчет параметров антенны. Продемонстрированы результаты использования антенны в лабораторных исследованиях. Показано, что наиболее высокочастотная часть спектра радиоизлучения приходится на предпробойную стадию разряда до начала резкого роста тока.
\end{abstract}

Ключевые слова: антенна Вивальди, микрополосковая антенна, радио излучение, моделирование и расчет антенны, атмосферная искра.

DOI: 10.21883/JTF.2021.12.51756.12-21

\section{Введение}

Исследование процессов в атмосферном разряде, приводящих к генерации различных типов излучений (рентгеновское, СВЧ, нейтронное идр.) важно для развития фундаментальной и прикладной физики газового разряда. Особый интерес представляет генерация СВЧ излучения. Комплексное исследование этого явления позволит лучше понять процессы, протекающие на начальной стадии разряда, в первые наносекунды после подачи высокого напряжения на электроды. Разработка методов анализа этого излучения позволит создать, в частности, грозопеленгаторы нового типа, работающих в диапазоне от сотен $\mathrm{MHz}$ до десяти $\mathrm{GHz}$ в отличие от современных образцов $(10-60 \mathrm{kHz})$. Это позволит точнее определять области грозовой активности, что имеет большое значение, например, в безопасности авиасообщений.

Генерация радиочастотного излучения во время формирования естественных молний на частотах более $500 \mathrm{MHz}$ были впервые зарегистрированы Такаги и Такеути в 1963 г. [1], а затем Бруком и Китагавой в 1964г. [2], которые сообщили о наблюдении радиоизлучений на частоте $0.85 \mathrm{GHz}$ с полосой $200 \mathrm{kHz}$, утверждая, что наблюдаемые излучения совпадают с отрицательным ступенчатым лидером, обратным ударом, молниевым лидером. В настоящий момент ведутся натурные измерения радиоизлучений от отрицательных ступенчатых лидеров молний в диапазоне $1.5-1.6 \mathrm{GHz}$ [3]. Излучение наблюдалось с помощью керамической микрополосковой антенны и цифрового радиоприемника, настроенного на центральную частоту $1.63 \mathrm{GHz}$ с полосой рабочих частот $2 \mathrm{MHz}$.

В лабораторных условиях исследованием данной проблемы занимаются и в наши дни. В работах $[4,5]$ авторам удалось зарегистрировать импульсы радиоизлучения частотой $2.4 \mathrm{GHz}$, максимум мощности которых совпадал с моментом генерации рентгеновского излучения (РИ). Максимумы высокочастотного радиоизлучения, как и импульсы РИ [6], наблюдались и в разрядах без пробоя (незавершенных разрядах), что может указывать на схожесть процессов их генерации.

Проводимое авторами [7] численное моделирование распространения и слияния стримеров в молниях в случае надпробойного поля $E_{0}=(1.5-2) \cdot E_{k}$, где $E_{k}-$ поле пробоя, показало возможность эффективной генерации электромагнитного излучения в диапазоне от десяти $\mathrm{MHz}$ до единиц $\mathrm{GHz}$ и выше. И если излучение порядка десяти $\mathrm{MHz}$, обычно используемое в системах грозопеленгации, связывается с уже известными физическими процессами распространения стримеров, возникающих в короне ступенчатого лидера при пробое чистого воздуха, то радиоизлучение в диапазоне от сотен $\mathrm{MHz}$ до десятка $\mathrm{GHz}$ может дать встречное столкновение стримеров за счет быстрого нарастания тока.

Аналогичные результаты по генерации радиоизлучения в диапазоне от десятков $\mathrm{MHz}$ до нескольких единиц $\mathrm{GHz}$ получены на стримерных столкновениях при нормальном атмосферном давлении в случае подпробойного поля $E_{0}<E_{k}[8]$.

При этом обычные экспоненциально растущие стримеры могут эффективно генерировать радиоизлучение в диапазоне $3-300 \mathrm{MHz}$ [9], но излучение на более 
$a$

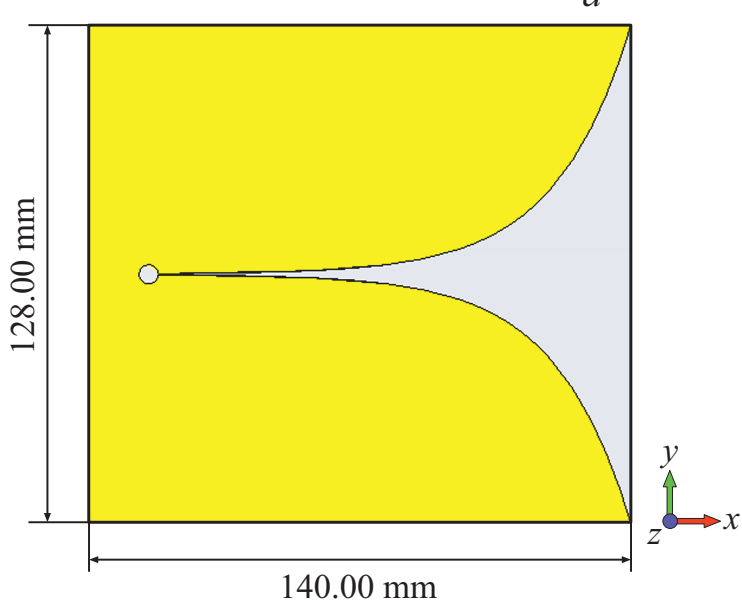

$b$

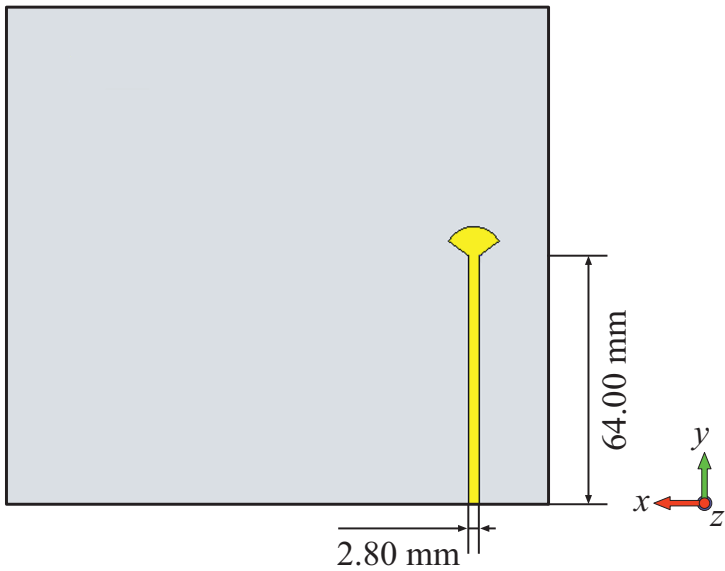

Рис. 1. Вид расчетной модели антенны с размерами: $a-$ „лицевая“ сторона с рабочей зоной и резонатором, $b-$ „оборотная“ сторона с микрополосковой линией.

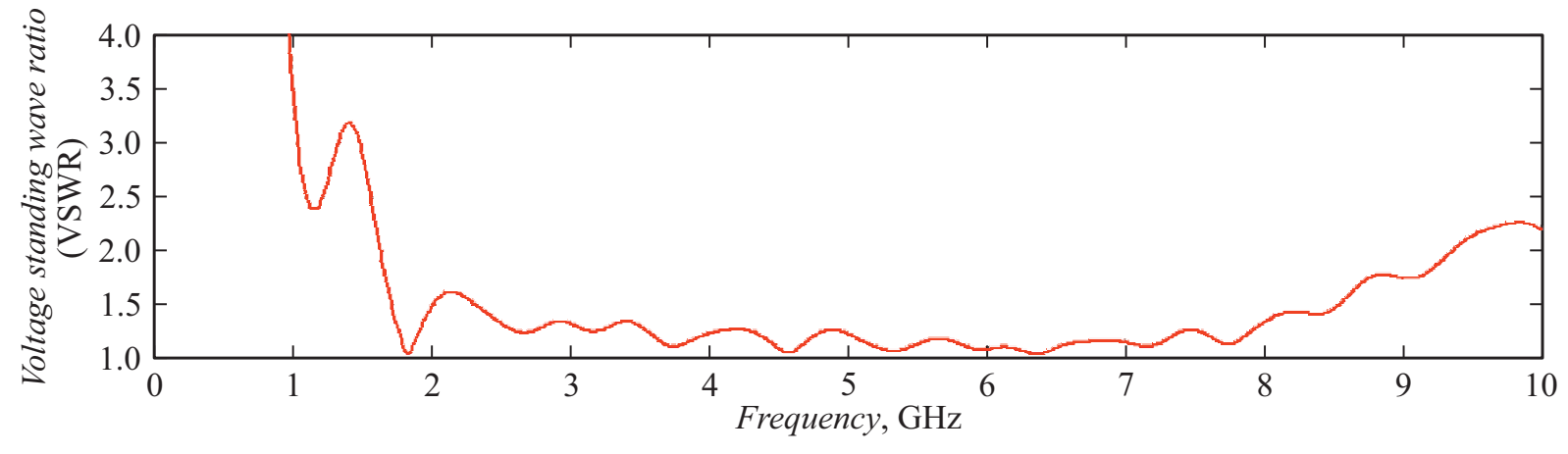

Рис. 2. Коэффициент стоячей волны по напряжению.

высоких частотах пренебрежимо мало, так как постоянная времени их роста - порядка наносекунд. Поэтому важно правильно идентифицировать процессы, которые могут приводить к очень быстрым изменениям тока, необходимым для генерации радиоизлучения соответствующих частотных диапазонов.

К сожалению, исследование генерации широкополосных радиоизлучений во время развития атмосферных разрядов в природе представляется достаточно сложной задачей. Грозовая активность проходит в течение лишь нескольких недель в году. При этом для набора достаточной статистики регистрирующую аппаратуру необходимо располагать максимально близко к грозовому фронту, который непрерывно движется. В качестве отдельной проблемы можно выделить исследование радиоизлучения сантиметрового диапазона и короче ввиду особенностей распространения этих волн в среде.

Тем не менее можно предположить, что процессы в разряде, приводящие к генерации соответствующих излучений, носят фундаментальный характер. В этом плане исследование формирования сопровождающихся эмиссией СВЧ разрядов в малых воздушных промежутках может пролить свет на механизмы генерации других сопутствующих излучений. В настоящей работе на примере разрядов в малых промежутках была исследована генерация СВЧ в лабораторном искровом разряде с $5 \mathrm{~mm}$ зазором между электродами, имеющими форму игла-плоскость. Приводятся спектры радиоизлучений, снятые на различных стадиях формирования искрового разряда. По ним видно, что разработанная антенна подходит для анализа физических процессов, генерирующих СВЧ излучение в лабораторной искровой плазме, и в будущем поможет сделать выводы о природе этих явлений.

\section{1. Аппаратура наблюдения}

Для регистрации радиоизлучения, генерирующегося в начальной фазе высоковольтного искрового разряда, была разработана сверхширокополосная антенна, построенная на основе расширяющейся щелевой линии (антенны Вивальди). Антенна Вивальди (АВ) копланарного типа (рис. 1) представляет собой диэлектрическую подложку, одна из сторон которой покрыта слоем металлизации в форме расширяющейся щели. Этот тип антенн 

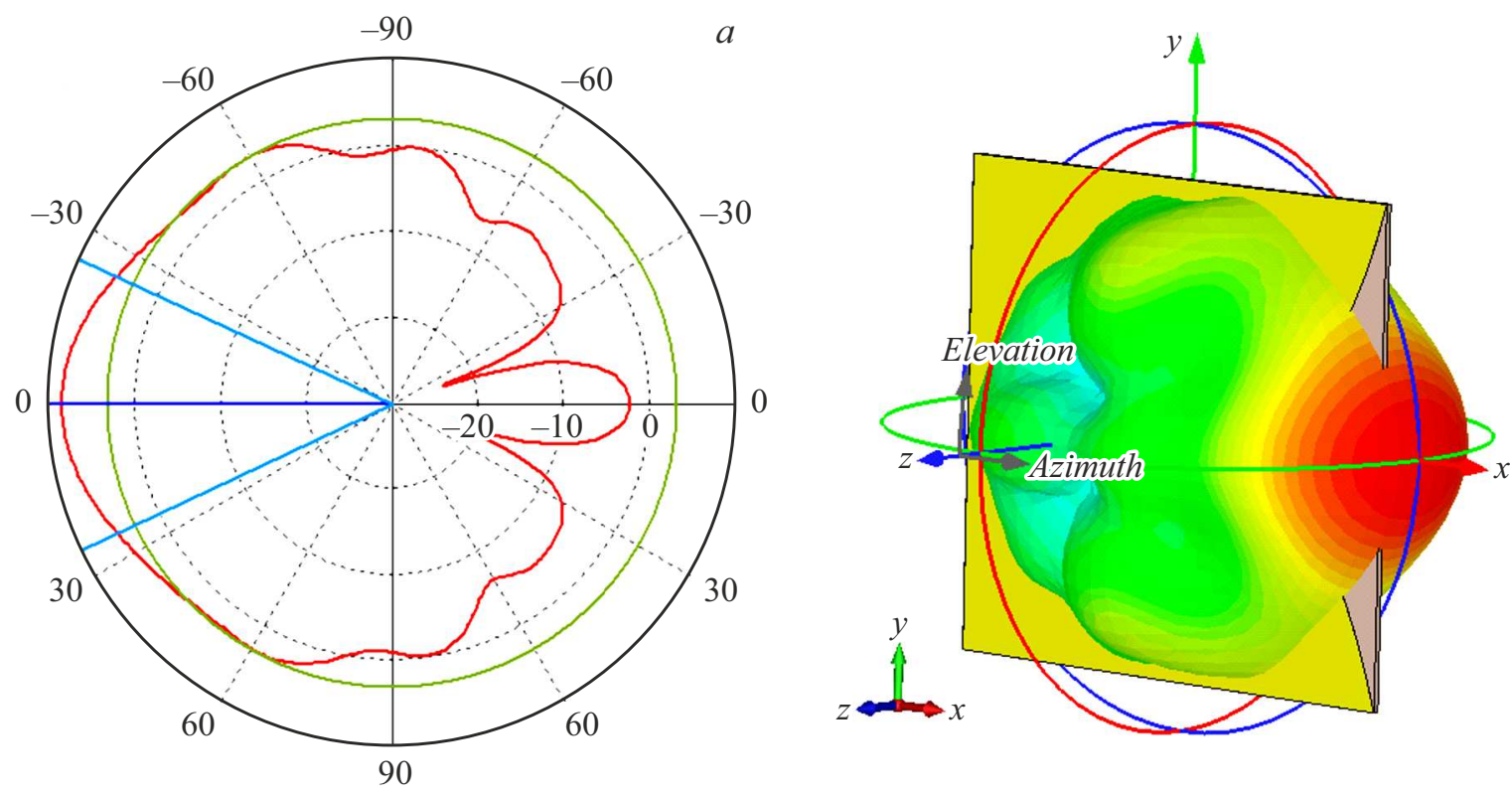

$b$

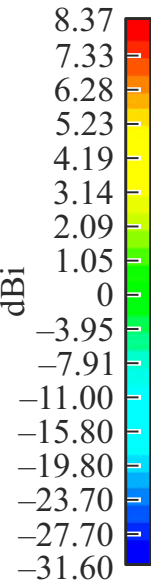

Рис. 3. Диаграмма направленности на частоте $3.5 \mathrm{GHz}: a-$ в плоскости $X Z, b-3 D$.

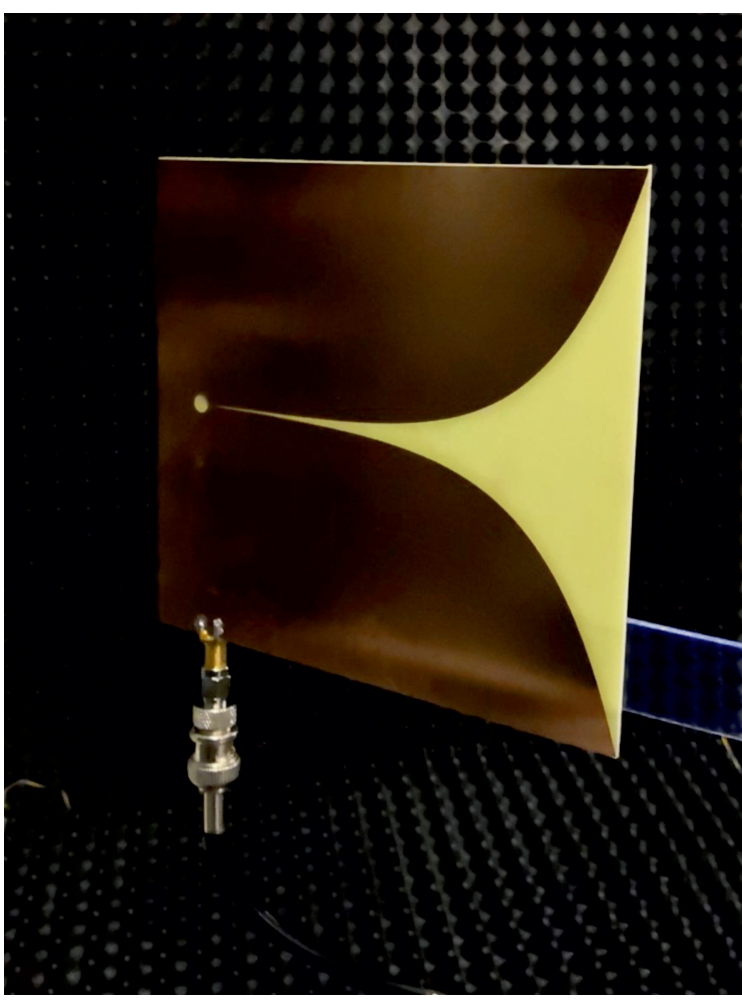

Рис. 4. Внешний вид разработанной АВ.

был выбран по ряду причин: широкий частотный диапазон (полоса рабочих частот), простота расчетов и изготовления, малые габариты. Малое усиление (порядка 6-8 dB) можно компенсировать установкой антенны на расстоянии 3-5 $\mathrm{m}$ от разряда. Это позволит находиться уже в „дальней зоне“, при этом мощность радиоизлу-
Характеристики разработанной АВ

\begin{tabular}{c|c}
\hline Рабочий диапазон частот & $1.66-9.36 \mathrm{GHz}$ \\
\hline Поляризация & Линейная \\
\hline $\begin{array}{c}\text { Коэффициентстоячей волны } \\
\text { в диапазоне рабочих частот }\end{array}$ & Нолее 2.0 \\
\hline Коэффициент усиления & $\begin{array}{l}\text { Не менее } 6 \mathrm{~dB} \text { с неравно- } \\
\text { мерностью не более } 2 \mathrm{~dB} \\
\text { конусе рабочих углов } \pm 25^{\circ}\end{array}$ \\
\hline Рабочий диапазон температур & От $-50^{\circ} \mathrm{C}$ до $+80^{\circ} \mathrm{C}$ \\
\hline Габариты & $140 \times 128 \times 1.55 \mathrm{~mm}$
\end{tabular}

чения останется достаточной для его регистрации этим типом антенн.

Моделирование и расчет параметров антенн производились в пакете программ CST microwave studio. Конструктивно антенны выполнены в виде медных печатных проводников на стеклотекстолите марки FR-4 толщиной $1.5 \mathrm{~mm}$. Материал обладает коэффициентом диэлектрической проницаемости $\varepsilon=4.5$ (измерено на $1 \mathrm{MHz}$ ), а также хорошими прочностными и стоимостными характеристиками. Толщина медного слоя с каждой стороны составляет $45 \mu \mathrm{m}$. К недостаткам FR-4 можно отнести тангенс угла диэлектрических потерь, который достигает значения 0.025 на частотах порядка $1 \mathrm{GHz}$ и выше. Это негативно сказывается на коэффициенте усиления. Но потери в диэлектрике учитываются при моделировании и расчете антенны, и с их учетом мы получаем характеристики антенны, удовлетворяющие требованиям проводимых экспериментов.

На основе анализа работ [10,11], раскрыв АВ, выполнен в виде расширяющейся по экспоненциальному 

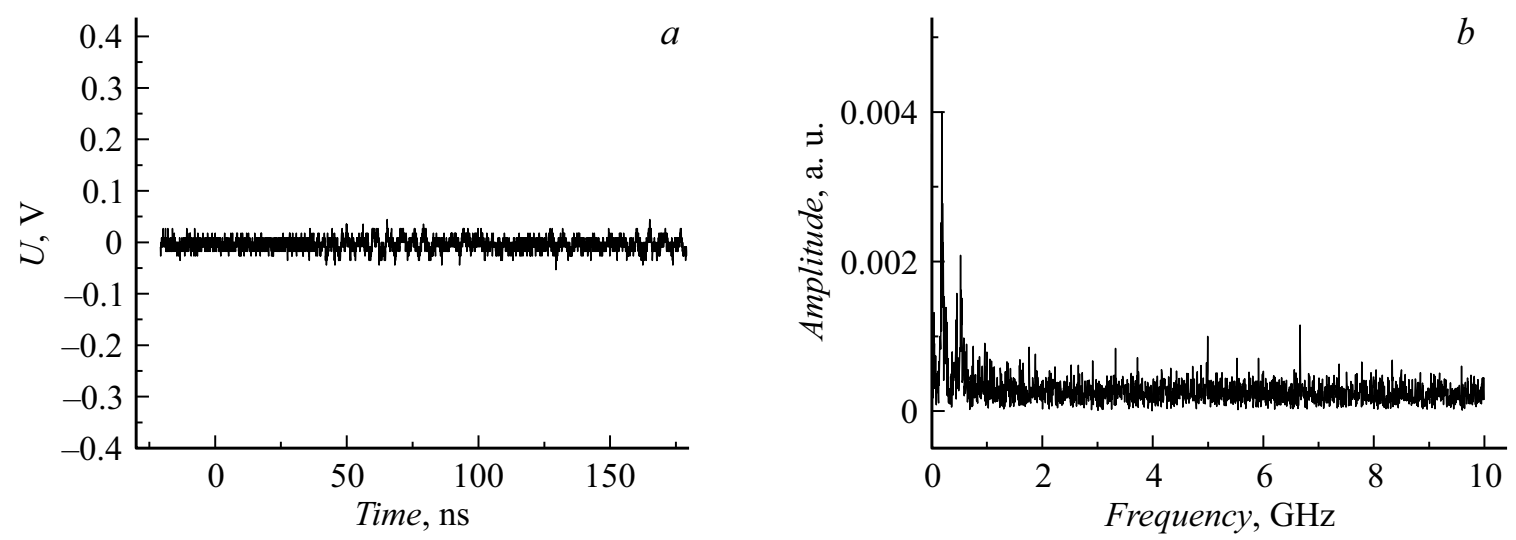

Pис. 5. $a-$ пример полученного с антенны сигнала в отсутствие протекания основного тока разряда, $b-$ спектр этого сигнала.

закону щели, $U_{y}=s \exp (r x)$, где $s=0.25 \mathrm{~mm}$ - это ширина щели в ее основании, $x$ - координата вдоль оси антенны, $k=0.05198-$ коэффициент кривизны, значение которого подбиралось эмпирически.

Согласование волнового сопротивления щели $\mathrm{AB}$ с 50-омным передающим кабелем осуществляется за счет микрополосковой линии. Расчет удовлетворяющих нас параметров импеданса и согласования микрополосковой линии с щелью антенны в точке возбуждения производился с помощью стандартной функции, встроенной в используемую расчетную программу. Для улучшения параметров согласования использовалось расширение на конце микрополосковой линии и резонатор диаметром $10 \mathrm{~mm}$ с ,лицевой“ стороны антенны.

Моделирование вариантов различных геометрий антенны проводилось в несколько итераций с увеличивающейся точностью и плотностью вычислительной сетки, что показало сходимость результатов при уменьшении размера ячейки. Представленные ниже результаты выполнены для сетки, разбитой с параметрами $699 \times 674 \times 46$ ячеек. Оптимизированный вариант имеет расчетный коэффициент стоячей волны, необходимый в лабораторных измерениях в диапазоне частот от 1.66 до $9.36 \mathrm{GHz}$ (рис. 2). Диаграмма направленности антенны показана на рис. 3.

Антенна была изготовлена путем фрезерования фольгированного стеклотекстолита на ЧПУ-станке. Для снятия сигнала с антенны используется разъем типоразмера SMA (рис. 4). Основные характеристики антенны приведены в таблице.

\section{2. Верификация}

Разработанная антенна использовалась для регистрации радиоизлучения в лабораторном искровом разряде с $5 \mathrm{~mm}$ зазором между электродами, имеющими форму игла-плоскость.

Антенна была установлена на расстоянии трех метров от лабораторной искры. Радиосигнал от антенны регистрировался осциллографом LeCroy WM 8620A

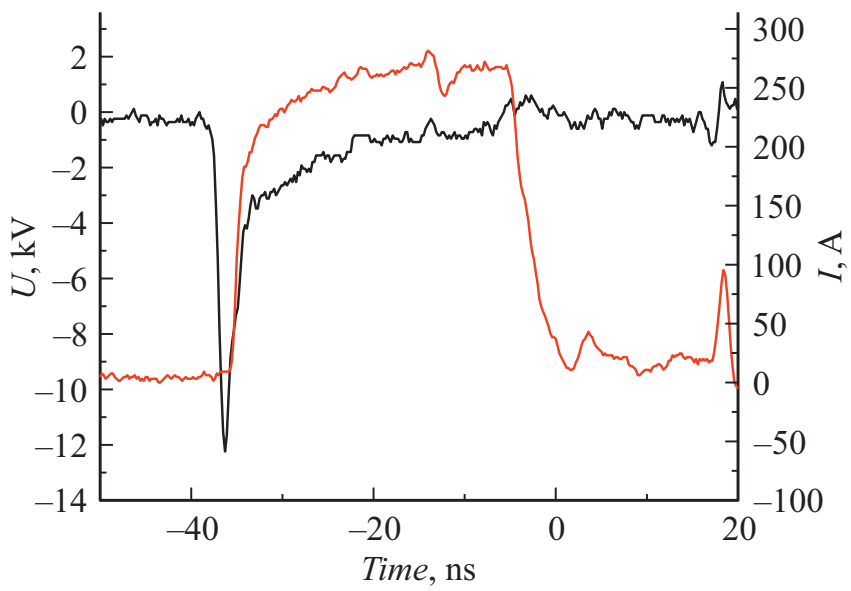

Рис. 6. Характеристики разряда: напряжение (слева, черный) и ток (справа, красный).

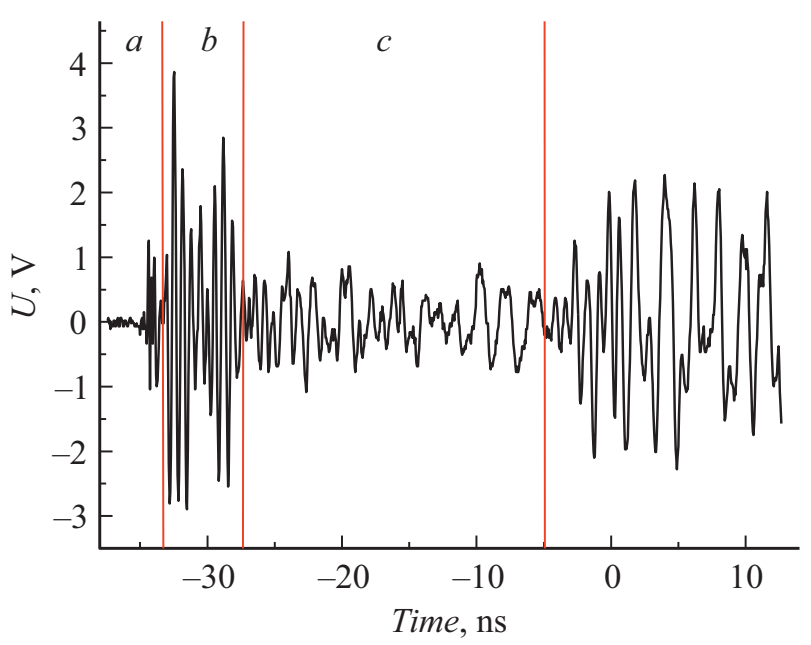

Рис. 7. Пример полученного с антенны сигнала: $a-$ предпробойная стадия, $b-$ стадия пробоя, $c-$ стадия протекания основного тока разряда.

с полосой пропускания $6 \mathrm{GHz}$. Передача радиосигнала до регистрирующей аппаратуры осуществляется по 

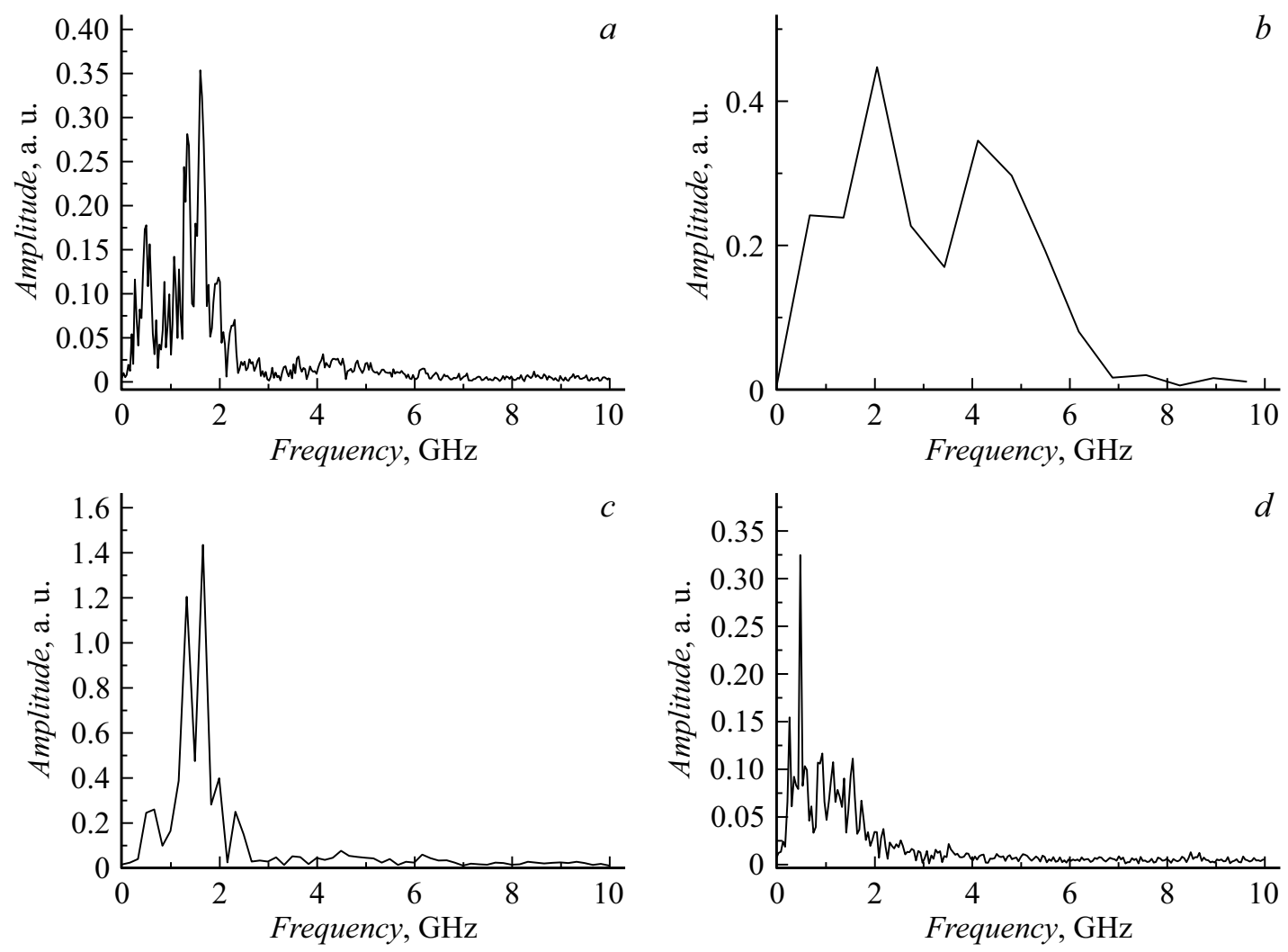

Рис. 8. Спектры радиоизлучения: $a-$ общий спектр всего разряда, $b-$ первая зона, с - вторая зона, $d-$ третья зона.

коаксиальному кабелю марки LMR-400 длиной $3 \mathrm{~m}$. Данный тип кабеля отличается от аналогов хорошим экранированием (не менее $90 \mathrm{~dB}$ ) и низкими уровнями потерь в кабеле: для частоты $1.5 \mathrm{GHz}-0.168 \mathrm{~dB} / \mathrm{m}$; для $1.8 \mathrm{GHz}-0.186 \mathrm{~dB} / \mathrm{m}$; для $2.0 \mathrm{GHz}-0.196 \mathrm{~dB} / \mathrm{m}$; для $2.5 \mathrm{GHz}-0.222 \mathrm{~dB} / \mathrm{m}$; для $5.8 \mathrm{GHz}-0.355 \mathrm{~dB} / \mathrm{m}$. Таким образом, потери в кабеле в диапазоне рабочих частот антенны не превышают $1-2 \mathrm{~dB}$.

Для оценки влияния электромагнитных наводок на кабель, а также влияния эховых условий в лаборатории и вторичных сигналов, принятых антенной, была поставлена серия экспериментов с подачей импульсного напряжения на высоковольтный электрод без нагрузки (в режиме „холостого хода“). В этой конфигурации видно, что при работе всей аппаратуры и генератора напряжения в отсутствии протекания основного тока разряда высокочастотное радиоизлучение отсутствует (рис. 5).

В эксперименте с высоковольтным разрядом в промежутке игла-плоскость длиной $5 \mathrm{~mm}$ с типичной вольтамперной характеристикой, показанной на рис. 6, с помощью разработанной АВ обнаружена генерация ВЧрадиоизлучения. Пример регистрируемых радиосигналов представлен на рис. 7. Амплитудный спектр радиосигнала, полученный при помощи быстрого фурьепреобразования (FFT), представлен на рис. 8, $a$.

В полученном сигнале можно выделить три характерные временные зоны, соответствующие разным стадиям развития разряда в исследуемом промежутке. Первая зона - предпробойная стадия (до начала резкого роста тока), характеризуется приложенным высоким напряжением. В этой стадии в ходе взрывной эмиссии появляются лавины электронов и формируются стримеры. Развиваясь, они начинают ионизовать среду по пути своего прохождения. В этой стадии можно наблюдать всплеск наиболее высокочастотной составляющей спектра радиоизлучения (до $6 \mathrm{GHz}$, рис. $8, b$ ). Вторая зона характеризуется падением напряжения и ростом тока это стадия развития сильноионизованной искры [12] после момента электрического пробоя промежутка. Спектр радиоизлучения на этой стадии разряда представлен на рис. 8,c. Амплитуда сигнала возрастает в сравнении с предпробойной стадией, при этом основной вклад вносит высокочастотное излучение на частотах $1.4-1.8 \mathrm{GHz}$, что говорит о сложности процессов формирования результирующей искры. Процессы плазмообразования начинают включать в себя, помимо ионизации среды лавинами электронов, быстрое нарастание проводимости в растущем канале, состоящем из сильноионизованных филаментов [12]. Третья зона - это стадия протекания основного тока разряда, когда электроды соединены результирующим искровым каналом. Характеризуется высокой интенсивностью излучения в диапазоне 200-500 MHz, спектр этой зоны представлен на рис. $8, d$. Возможно, это связано с плазменными колебаниями в сильноионизованной искре. 


\section{Заключение}

В работе предложены расчет, моделирование и разработка сверхширокополосной антенны с расширяющейся щелью для применения в экспериментах по регистрации радиоизлучения, генерирующегося в начальной фазе искрового разряда. Изготовлена антенна с рабочим диапазоном частот $1.66-9.36 \mathrm{GHz}$ с коэффициентом стоячей волны по напряжению, не превышающим 2.0. Приведены результаты использования антенны в лабораторных исследованиях. Получены спектры радиоизлучений различных стадий формирования искрового разряда.

Анализ полученного спектра радиоизлучения позволяет выделить различные стадии развития искрового разряда. Дальнейшая разработка антенн поможет в получении количественной информации о процессах в плазме. Результаты в первом приближении согласуются с экспериментальными и аналитическими работами по исследованию грозовых и лабораторных искровых разрядов.

\section{Финансирование работы}

Работа выполнена при поддержке гранта РФФИ, проект № 20-08-01156.

\section{Конфликт интересов}

Авторы заявляют, что у них нет конфликта интересов.

\section{Список литературы}

[1] M. Takagi, T. Takeuti. Proceed. Res. Institute Atmospherics, Nagoya Univer., 10, (1963).

[2] M. Brook, N. Kitagawa. J. Geophys. Res., 69(12), 2431 (1964).

[3] D. Petersen, W. Beasley. Atmos. Res., 135, 314 (2014).

[4] J. Montanya, F. Fabry, V. March, O. van der Velde, G. Sola, D. Romero. J. Atmospheric Solar-Terrestrial Phys., 136, 94 (2015).

[5] P. Kochkin, J. Montanya, V. March. Techniq. Measurements, 1, 157 (2016)

[6] Y.L. Stankevich, V.G. Kalinin. Soviet Phys. Dok1., 12, 1042 (1968).

[7] F. Shi, N. Liu, J.R. Dwyer, K.M.A. Ihaddadene. Geophys. Res. Lett., 46, 443 (2018)

[8] A. Luque. J. Geophys. Res.: Atmospheres, 122, 10497 (2017).

[9] F. Shi, N.Y. Liu, H.K. Rassoul. J. Geophys. Res.: Atmospheres, 121, 7284 (2016).

[10] И.Г. Рязанов, А.А. Бякин, О.А. Белоусов. Вопросы современной науки и практики. Ун-т им. В.И. Вернадского, 2, 297 (2013).

[11] П.Д. Куроптев, В.В. Левяков, А.В. Фатеев. Доклады Томского гос. ун-та систем управления и радиоэлектроники, 19 (2), 23 (2016).

[12] E.V. Parkevich, M.A. Medvedev, G.V. Ivanenkov, A.I. Khirianova, A.S. Selyukov, A.V. Agafonov, Ph.A. Korneev, S.Y. Gus'kov, A.R. Mingaleev. Plasma Sourc. Sci. Technol., 28 (9), 095003 (2019). 